\title{
Several Topological Indices of Two Kinds of Tetrahedral Networks
}

\author{
Jia-Bao Liu (iD) and Lu-Lu Fang $(D)$ \\ School of Mathematics and Physics, Anhui Jianzhu University, Hefei 230601, China \\ Correspondence should be addressed to Jia-Bao Liu; liujiabaoad@163.com and Lu-Lu Fang; fangluluajd@163.com
}

Received 29 July 2021; Accepted 1 September 2021; Published 28 September 2021

Academic Editor: Ljubisa Kocinac

Copyright (c) 2021 Jia-Bao Liu and Lu-Lu Fang. This is an open access article distributed under the Creative Commons Attribution License, which permits unrestricted use, distribution, and reproduction in any medium, provided the original work is properly cited.

\begin{abstract}
Tetrahedral network is considered as an effective tool to create the finite element network model of simulation, and many research studies have been investigated. The aim of this paper is to calculate several topological indices of the linear and circle tetrahedral networks. Firstly, the resistance distances of the linear tetrahedral network under different classifications have been calculated. Secondly, according to the above results, two kinds of degree-Kirchhoff indices of the linear tetrahedral network have been achieved. Finally, the exact expressions of Kemeny's constant, Randic index, and Zagreb index of the linear tetrahedral network have been deduced. By using the same method, the topological indices of circle tetrahedral network have also been obtained.
\end{abstract}

\section{Introduction}

In actual life, many problems can be described using graph models. The graph model as a tool to describe network has been widely studied. Each network can be considered as graph. The problems of the vertices in the graph correspond to the points in the network, and the edges in the graph correspond to the network connection relationship between the points. In this paper, only simple, undirected, and connected graphs are considered. Suppose $G=(V(G), E(G))$ is a graph, and it satisfies $|V(G)|=n$ and $|E(G)|=m$. The degree of vertex $p$ in the graph $G$ is denoted by $d_{p}$. Connecting the two vertices $p, q \in V(G)$, the distance $d_{G}(p, q)[1]$ is defined as the length of the shortest path. And the resistance distance between vertex $p$ and vertex $q$ is delimited as the effective resistance, which is denoted as $r_{G}(p, q)$ [2]. For more terminologies, one can refer to reference [3].

The sum of the resistance distance between each pair of vertices in the graph $G$ is defined as the Kirchhoff index [2], as follows:

$$
K f(G)=\sum_{p, q \in V(G)} r_{G}(p, q)
$$

Similarly, Chen and Zhang [4] put forward the following definition of the multiplicative degree-Kirchhoff index, that is, as follows:

$$
K f^{*}(G)=\sum_{p, q \in V(G)} d_{p} d_{q} r_{G}(p, q) .
$$

Subsequently, Gutman et al. [5] proposed the following definition of the additive degree-Kirchhoff index, that is, as follows:

$$
K f^{+}(G)=\sum_{p, q \in V(G)}\left(d_{p}+d_{q}\right) r_{G}(p, q) .
$$

For a random walk $[6,7]$ in a network, the expectation of the average first arrival time $[8,9]$ from a vertex $p$ to another vertex $q$ selected according to the stable distribution of Markov process [10-13] is called Kemeny's constant of the network. Kemeny's constant is given by

$$
K(G)=\frac{1}{4 a} \sum_{p, q \in V(G)} d_{p} d_{q} r_{G}(p, q),
$$

where $a$ is the number of edges in the graph $G$.

In previous studies, several topological indices based on vertex-degree have been applied in research. The following 
three topological indices (Kemeny's constant, Randic index, and Zagreb index) are the most widely used:

$$
\begin{gathered}
M_{1}=M_{1}(G)=\sum_{p \in V(G)}\left(d_{p}\right)^{2}, \\
M_{2}=M_{2}(G)=\sum_{p q \in E(G)} d_{p} d_{q}, \\
R=R(G)=\sum_{p q \in E(G)} \frac{1}{\sqrt{d_{p} d_{q}}} .
\end{gathered}
$$

By the definition of the multiplicative (the additive) degree-Kirchhoff index, the main job is to calculate $r_{G}(p, q)$. From the perspective of practical application, the resistance distance considers all the paths between any two vertices, not just the short path, so the resistance distance can reflect the relationship between any two vertices better than the distance. This paper applies the expressions of the resistance distance between any two vertices of the linear and circle tetrahedral networks to derive the multiplicative (the additive) degree-Kirchhoff index of them, respectively. This kind of linear tetrahedral network is a one-dimensional infinitely extended network which is linked by a series of tetrahedrons. Its structure is morphologically manifested as elongation in one direction (see Figure 1), and $n$ is the number of the tetrahedron in the network. The structure of this kind of circle tetrahedral network is a combination of tetrahedrons and octahedrons whose form is a two-dimensional wireless extension. And the octahedrons are connected by common edge (see Figure 2).

The structure of this paper is shown as follows: we introduce several fundamental definitions of the electrical network and give two important lemmas in Section 2. We present some proofs of our main results, namely, the multiplicative (the additive) degree-Kirchhoff index, Kemeny's constant [14-16], Randic index [17-20], and Zagreb index $[21,22]$ of the linear and circle tetrahedral networks in Section 3. We summarize this article in Section 4 .

\section{Preliminaries}

In the following section, we will give two important lemmas that will make a tremendous effect on our conclusions.

Lemma 1 (see [23]). Suppose the distance between vertex $p$ and vertex $q$ is $t$ and $p, q \in L(n),(n \geq 3)$.

$$
r_{G}(p, q)=\frac{t}{2} \text {. }
$$

Lemma 2 (see [23]). Suppose the distance between vertex $p$ and vertex $q$ is $t$ and $p, q \in C(n),(n \geq 3)$.

(1) $r_{G}(p, q)=(t(n-t) / 2 n)$ when $d_{p}=d_{q}=6$ and $1 \leq t \leq\lfloor n / 2\rfloor$

(2) $r_{G}(p, q)=((2 t-1)(2 n-2 t+1) / 8 n)+(1 / 4)$ when $d_{p}=3, d_{q}=6$, and $1 \leq t \leq\lfloor(n+1) / 2\rfloor$

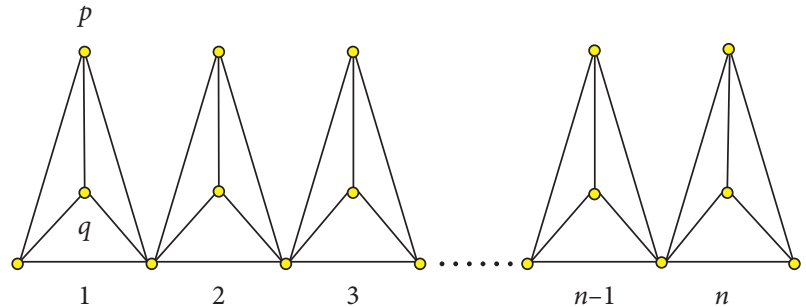

Figure 1: A kind of linear tetrahedron $L(n)$.

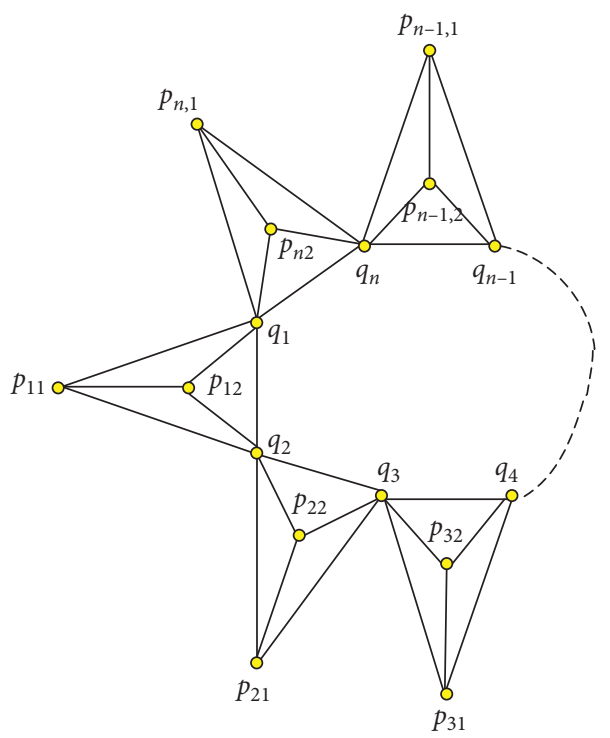

FIgURE 2: A kind of circle tetrahedron $C(n)$.

(3) $r_{G}(p, q)=((t-1)(n-t+1) / 2 n)+(1 / 2)$ when $d_{p}=$ $d_{q}=3$ and $1<t \leq\lfloor(n / 2)+1\rfloor$

(4) $r_{G}(p, q)=(1 / 2)$ when $d_{p}=d_{q}=3$ and $t=1$

\section{Main Results}

In this section, the main purpose is to derive the multiplicative (the additive) degree-Kirchhoff index, Kemeny's constant, Randic index, and Zagreb index of the linear (the circle) network (see Figures 3 and 4).

\subsection{The Linear Tetrahedral Network}

\subsubsection{The Additive Degree-Kirchhoff Index}

Theorem 1. Let $L(n),(n \geq 3)$ be a linear tetrahedral network.

$$
K f^{+}(L(n))=\frac{12 n^{3}+27 n^{2}-3 n}{2} .
$$

Proof. The linear tetrahedral network $L(n)$ is shown in Figure 3 . The number of vertices in $L(n)$ is $3 n+1$, and the number of edges in $L(n)$ is $6 n$. When the distance between any two vertices is 1 , the number of pairs of degree three and 


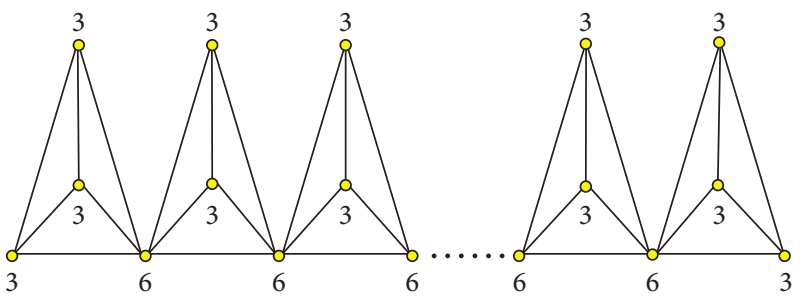

Figure 3: The linear tetrahedral network $L(n)$.

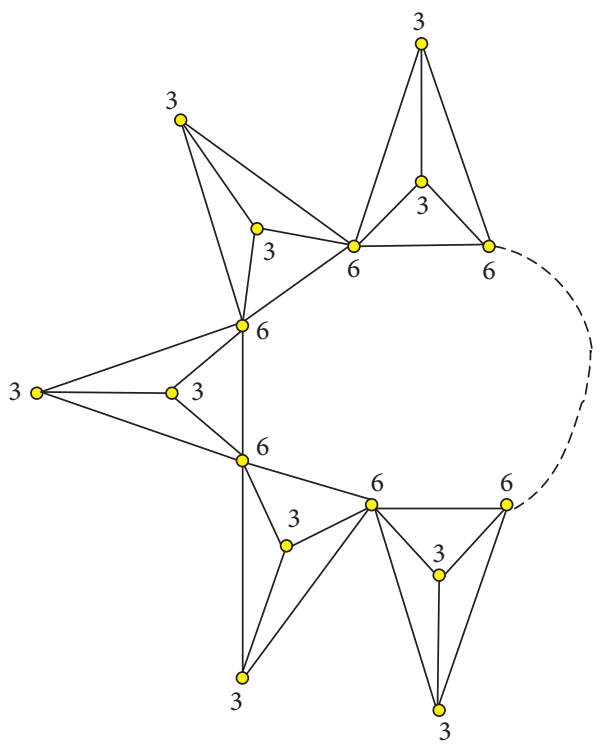

and degree six is $n-2$. Besides, the maximum distance between the vertices of degree three and degree three in $L(n)$ is $n$. The maximum distance between the vertices of degree three and degree six in $L(n)$ is $n-1$. The maximum distance between the vertices of degree six and degree six in $L(n)$ is $n-2$. When the distance between any two vertices is $t$, there are $4[n-(t+1)]+12$ pairs of degree three and degree three and $4[n-(t+1)]+6$ pairs of degree three and degree six, where $t=2,3, \ldots, n-1$. And when the distance between any two vertices is $t$, the number of pairs of degree six and degree six is $n-(t+1)$, where $t=2,3, \ldots, n-2$. Specially, when $t=n$, there are 9 pairs of vertices between degree three and degree three. The above contents are shown in Table 1.

By using equation (3) and Lemma 1, we calculate the following result:

FIgURE 4: The circle tetrahedral network $C(n)$.

degree three is $n+4$, the number of pairs of degree three and degree six is $4 n-2$, and the number of pairs of degree six

$$
\begin{aligned}
K f^{+}(L(n))= & {\left[\frac{(3+3)(n+4)+(3+6)(4 n-2)+(6+6)(n-2)}{2}\right] } \\
& +\left[\sum_{t=2}^{n-2} \frac{[n-(t+1)] t}{2} \times(6+6)+\sum_{t=2}^{n-1} \frac{t[4(n-(t+1))+12]}{2} \times(3+3)\right. \\
& \left.+\frac{9 n}{2} \times(3+3)+\sum_{t=2}^{n-1} \frac{t[4(n-(t+1))+6]}{2} \times(3+6)\right] \\
= & (54 n-9)+\left[-36 \sum_{t=2}^{n-1} t^{2}+(36 n+27) \sum_{t=2}^{n-1} t\right] \\
= & (54 n-9)+\left[-36 \frac{(n-1)(n-1+1)[2(n-1)+1]}{6}+36\right]+(36 n+27)\left[\frac{[2+(n-1)](n-2)]}{2}\right] \\
= & \frac{12 n^{3}+27 n^{2}-3 n}{2} .
\end{aligned}
$$


TABLe 1: The classification of edge-pairs of $C(n)$.

\begin{tabular}{lccc}
\hline$\left(d_{p}, d_{q}\right)$ & $(3,3)$ & $(6,6)$ & $(3,6)$ \\
\hline Number of the edges $(d=1)$ & $n+4$ & $n-2$ & $4 n-2$ \\
Number of the edges $(d=t)$ & $4[n-(t+1)]+12$ & $n-(t+1)$ & $4[n-(t+1)]+6$ \\
\hline
\end{tabular}

Then, we obtain our desired consequence.

$$
K f^{*}(L(n))=12 n^{3}+18 n^{2}-3 n
$$

3.1.2. The Multiplicative Degree-Kirchhoff Index. As shown above, we can find the multiplicative degree-Kirchhoff index of the linear network.
Proof. By using equation (2) and Lemma 1, we calculate the following result:

Theorem 2. . Let $L(n),(n \geq 3)$ be a linear tetrahedral network.

$$
\begin{aligned}
K f^{*}(L(n))= & \left.\frac{(3+3)(n+4)+(3+6)(4 n-2)+(6+6)(n-2)}{2}\right] \\
& +\left[\sum_{t=2}^{n-2} \frac{(n-(t+1)) t}{2} \times(6 \times 6)+\sum_{t=2}^{n-1} \frac{t[4(n-(t+1))+12]}{2} \times(3 \times 3)\right. \\
& \left.+\frac{9 n}{2} \times(3 \times 3)+\sum_{t=2}^{n-1} \frac{t[4(n-(t+1))+6]}{2} \times(3 \times 6)\right] \\
= & (99 n-36)+2\left[-36 \sum_{t=2}^{n-1} t^{2}+(36 n+18) \sum_{t=2}^{n-1} t\right] \\
= & (99 n-36)+2\left[-36 \frac{(n-1)(n-1+1)[2(n-1)+1]}{6}+36\right]+2(36 n+18)\left[\frac{[2+(n-1)](n-2)]}{2}\right] \\
= & 12 n^{3}+18 n^{2}-3 n .
\end{aligned}
$$

3.1.3. Kemeny's Constant, Randic Index, and Zagreb Index. The relationship between vertices and edges of $L(n)$ based on degree is shown in Tables 2 and 3.
By utilizing equations (4)-(7) and Tables 2 and 3, the following three indices can be achieved easily: 
TABLE 2: The classification of vertex-pairs of $L(n)$.

\begin{tabular}{lcc}
\hline Degrees & 3 & 6 \\
\hline Number of vertices & $2 n+2$ & $n-1$ \\
\hline
\end{tabular}

TABle 3: The classification of edge-pairs of $L(n)$.

\begin{tabular}{llll}
\hline$\left(d_{p}, d_{q}\right)$ & $(3,3)$ & $(6,6)$ & $(3,6)$ \\
\hline Number of edges & $n+4$ & $n-2$ & $4 n-2$ \\
\hline
\end{tabular}

$$
\begin{aligned}
M_{1}(L(n))= & (2 n+2) 3^{2}+(n-1) 6^{2}=54 n-17 \\
M_{2}(L(n))= & (n+4)(3 \times 3)+(n-2)(6 \times 6)+(4 n-2)(3 \times 6)=117 n-72, \\
R(L(n))= & (n+4) \frac{1}{\sqrt{3 \times 3}}+(n-2) \frac{1}{\sqrt{6 \times 6}}+(4 n-2) \frac{1}{\sqrt{3 \times 6}} \\
K(L(n))= & \frac{1}{4 \times 6 n}\left[\frac{(3+3)(n+4)+(3+6)(4 n-2)+(6+6)(n-2)}{2}\right. \\
& +\sum_{t=2}^{n-2} \frac{[n-(t+1)] t}{2} \times(6 \times 6)+\sum_{t=2}^{n-1} \frac{t[4(n-(t+1))+12]}{2} \times(3 \times 3) \\
& \left.+\frac{9 n}{2} \times(3 \times 3)+\sum_{t=2}^{n-1} \frac{t[4(n-(t+1))+6]}{2} \times(3 \times 6)\right] \\
= & \frac{4 n^{2}+6 n-1}{8} .
\end{aligned}
$$

\subsection{The Circle Tetrahedral Network}

\subsubsection{The Additive Degree-Kirchhoff Index}

Theorem 3. Let $C(n),(n \geq 3)$ be a circle tetrahedral network.

$$
K f^{+}(C(n))=\frac{12 n^{3}+42 n^{2}-15 n}{4} \text {. }
$$

Proof. The labels for all vertices are shown in Figure 2. In order to calculate conveniently, allow $p_{m}=p_{n}$ if $m \equiv n(\bmod s)$ and $p_{m k}=p_{n k}$ if $m \equiv n(\bmod s)(s=1,2)$. Set $p$ and $q$ as different vertices in $C(n)$. Results can be divided into the following six cases:

Case 1. $\sum_{\substack{\{p, q\} \subset V(G) \\ d_{p}=d_{q}=3}} r(p, q)$ for even $n$.
When $t=1$, the number of vertices of degree three in $L(n)$ is $n$. For every $p \in\left\{p_{m 1}, p_{m 2}\right\}(m=1,2, \ldots, n)$, if $2 \leq t \leq(n / 2)$, then $p$ must be a member of the set $\left\{p_{m+t-1,1}, p_{m+t-1,2}, p_{m-(t-1), 1}, p_{m-(t-1), 2}\right\}$, so when the distance between any two vertices is $p$, there are $((2 \times$ $4 \times n) / 2)=4 n$ pairs of them. For every $p \in\left\{p_{m 1}, p_{m 2}\right\}(m=1,2, \ldots, n)$, if $t=(n / 2)+1$, then $m$ must be a member of the set $\left\{p_{m+(n / 2), 1}, p_{m+(n / 2), 2}\right\}$ and thus when distance between any two vertices is $(n / 2)+1$, there are $((2 \times 2 \times n) / 2)=2 n$ pairs. Then, through Lemma 2 , we gain

$$
\begin{aligned}
\sum_{\substack{\{p, q\} \subset V(G) \\
d_{p}=d_{q}=3}}\left(d_{p}+d_{q}\right) r(p, q) & =\frac{3 n^{2}+18 n}{2}+12 \sum_{t=2}^{(n / 2)}[t(n-t+2)-1] \\
& =\frac{3 n^{2}+18 n}{2}+12 \sum_{t=2}^{(n / 2)}\left(n t-t^{2}+2 t-1\right) .
\end{aligned}
$$


Case 2. $\sum_{\substack{\{p, q\} \subset V(G) \\ d_{p}=d_{q}=3}}^{r(p, q) \text { for odd } n .}$

When $t=1$, the number of vertices of degree three in $L(n)$ is $n$. For each $p \in\left\{p_{m 1}, p_{m 2}\right\}(m=1,2, \ldots, n)$, if $2 \leq t \leq((n+1) / 2), \quad$ then $n \in\left\{p_{m+t-1,1}, p_{m+t-1,2}\right.$, $\left.p_{m-(t-1), 1}, p_{m+(t-1), 2}\right\}$, so when $d_{p q}=t$, the number of the pairs of the vertex $p$ and $q$ is $4 n$. Through Lemma 2, we gain

$\sum_{\{p, q\} \subset V(G)}\left(d_{p}+d_{q}\right) r(p, q)=3 n+12 \sum_{t=2}^{((n+1) / 2)}\left(n t-t^{2}+2 t-1\right)$. $d_{p}=d_{q}=3$

Case 3. $\sum_{\substack{\{p, q\} \subset V(G) \\ d_{p}=d_{q}=6}} r(p, q)$ for even $n$.

For each $p=q_{m}(m=1,2, \ldots, n)$, if $1 \leq t \leq(n / 2)$, then $p \in\left\{p_{m+t}, p_{m-t}\right\}$, and thus when $d_{p q}=t$, the number of the pairs of the vertex $p$ and $q$ is $n$. For every $p=q_{m}(m=1,2, \ldots, n), \quad$ if $t=(n / 2), \quad$ then $p \in\left\{p_{m+(n / 2)}\right\}$, and thus when $d_{p q}=(n / 2)$, there are $(n / 2)$ pairs. Then, by using Lemma 2 , we infer

$$
\begin{aligned}
& \sum_{\substack{\{p, q\} \subset V(G) \\
d_{p}=d_{q}=6}}\left(d_{p}+d_{q}\right) r(p, q)=\frac{3 n^{2}}{4}+6 \sum_{t=1}^{(n / 2)-1}[t(n-t)] \\
& =\frac{3 n^{2}}{4}+6 \sum_{t=1}^{(n / 2)-1}\left(n t-t^{2}\right) .
\end{aligned}
$$

Case 4. $\sum_{\substack{\{p, q\} \subset V(G) \\ d}} r(p, q)$ for odd $n$.

When $p=q_{m}(m=1,2, \ldots, n)$, if $1 \leq t \leq(n / 2)$, then $p \in\left\{p_{m+t}, p_{m-t}\right\}$, and thus when $d_{p q}=t$, there are $n$ pairs. Then, by using Lemma 2, we deduce

$$
\sum_{\{p, q\} \subset V(G)}\left(d_{p}+d_{q}\right) r(p, q)=6 \sum_{t=1}^{((n-1) / 2)}\left(n t-t^{2}\right) .
$$

Case 5. $\sum_{\{p, q\} \subset V(G)} r(p, q)$ for even $n$.

$$
d_{p}=3, d_{q}=6
$$

For each $p_{m 1}, p_{m 2}(m=1,2, \ldots, n)$, if $1 \leq t \leq(n / 2)$, then $p \in\left\{p_{m+t}, p_{m+1-t}\right\}$, and thus when $d_{p q}=t$, there are $4 n$ pairs. Then, by using Lemma 2 , we gain

$$
\begin{aligned}
\sum_{\substack{\{p, q\} \subset V(G) \\
d_{p}=3, d_{q}=6}}\left(d_{p}+d_{q}\right) r(p, q) & =9 \sum_{t=1}^{(n / 2)}\left[\frac{(2 t-1)(2 n-2 t+1)}{2}+n\right] \\
& =9 \sum_{t=1}^{(n / 2)}\left(2 n t-2 t^{2}+2 t-\frac{1}{2}\right) .
\end{aligned}
$$

Case 6. $\sum_{\substack{\{p, q\} \subset V(G) \\ d_{p}=3, d_{q}=6}} r(p, q)$ for odd $n$.

For every $\quad p_{m 1}, p_{m 2}(m=1,2, \ldots, n), \quad$ if $1 \leq t \leq((n-1) / 2)$, then $p \in\left\{p_{m+t}, p_{m+1-t}\right\}$, so when $d_{p q}=t$, the number of the pairs of the vertex $p$ and $q$ is $4 n$. For each $p_{m 1}, p_{m 2}(m=1,2, \ldots, n)$, if $t=((n+1) / 2)$, then $p$ must be $p_{m+(n+1 / 2)}$, and thus when $d_{p q}=((n+1) / 2)$, there are $2 n$ pairs. Then, by using Lemma 2 , we attain

$$
\sum_{\substack{\{p, q\} \subset V(G) \\ d_{p}=3, d_{q}=6}}\left(d_{p}+d_{q}\right) r(p, q)=\frac{9 n^{2}+18 n}{4}+9 \sum_{t=1}^{((n-1) / 2)}
$$

$$
\cdot\left(2 n t-2 t^{2}+2 t-\frac{1}{2}\right)
$$

If $n$ is even, by applying equations (15), (17), and (19) and Lemma 2, then

$$
\begin{aligned}
K f^{+}(C(n)) & =\frac{3 n^{2}-12 n}{4}+\sum_{t=1}^{(n / 2)}\left(36 n t-36 t^{2}+42 t-\frac{33}{2}\right) \\
& =\frac{3 n^{2}-12 n}{4}+\left[-36 \sum_{t=1}^{(n / 2)} t^{2}+36 n \sum_{t=1}^{(n / 2)} t+\sum_{t=1}^{(n / 2)}\left(42 t-\frac{33}{2}\right)\right] \\
& =\frac{3 n^{2}-12 n}{4}+\left[-36 \frac{(n / 2)((n / 2)+1)(n+1)}{6}+36 n \frac{(n / 2)((n / 2)+1)}{2}+\frac{(n / 2)(21 n+9)}{2}\right] \\
& =\frac{12 n^{3}+42 n^{2}-15 n}{4} .
\end{aligned}
$$


If $n$ is odd, by applying equations (16), (18), and (20) and Lemma 2, then

$$
\begin{aligned}
K f^{+}(C(n))= & \frac{21 n^{2}+30 n-12}{4}+\sum_{t=1}^{((n-1) / 2)}\left(36 n t-36 t^{2}+42 t-\frac{33}{2}\right) \\
= & \frac{21 n^{2}+30 n-12}{4}+\left[-36 \sum_{t=1}^{((n-1) / 2)} t^{2}+36 n \sum_{t=1}^{((n-1) / 2)} t+\sum_{t=1}^{((n-1) / 2)}\left(42 t-\frac{33}{2}\right)\right] \\
= & \frac{21 n^{2}+30 n-12}{4}+\left[-36 \frac{((n-1) / 2)(((n-1) / 2)+1)(n-1+1)}{6}+36 n \frac{((n-1) / 2)(((n-1) / 2)+1)}{2}\right. \\
& \left.+\frac{((n-1) / 2)(21 n-12)}{2}\right] \\
= & \frac{12 n^{3}+42 n^{2}-15 n}{4} .
\end{aligned}
$$

Consequently, the proof is completed.

3.2.2. The Multiplicative Degree-Kirchhoff Index. As shown above, the multiplicative degree-Kirchhoff index of the circle network can be derived by classification.

Theorem 4. Let $C(n),(n \geq 3)$ be a circle tetrahedral network.

$$
K f^{*}(C(n))=6 n^{3}+18 n^{2}-6 n .
$$

Proof. The results in six cases are given as follows:

Case 1. $\begin{gathered}\sum_{\{p, q\} \subset V(G)} r(p, q) \text { for even } n . \\ d_{p}=d_{q}=3\end{gathered}$

$$
\sum_{\substack{\{p, q\} \subset V \\ d_{p}=d_{q}=3}} d_{p} d_{q} r(p, q)=\frac{9 n^{2}+54 n}{4}+18 \sum_{t=2}^{(n / 2)}\left(n t-t^{2}+2 t-1\right) .
$$

Case 2. $\sum\{p, q\} \subset V(G) r(p, q)$ for odd $n$.

$$
d_{p}=d_{q}=3
$$

$\sum_{\{p, q\} \subset V} d_{p} d_{q} r(p, q)=\frac{9 n}{2}+18 \sum_{t=2}^{((n+1) / 2)}\left(n t-t^{2}+2 t-1\right)$.

$d_{p}=d_{q}=3$

Case 3. $\begin{gathered}\sum\{p, q\} \subset V(G) \\ \left\{\begin{array}{c} \\ d_{p}=d_{q}=6\end{array}\right.\end{gathered}$

$$
d_{p}=d_{q}=6
$$

$$
\sum_{\substack{\{p, q\} \subset V \\ d_{p}=d_{q}=6}} d_{p} d_{q} r(p, q)=\frac{9 n^{2}}{4}+18 \sum_{t=1}^{(n / 2)-1}\left(n t-t^{2}\right) .
$$

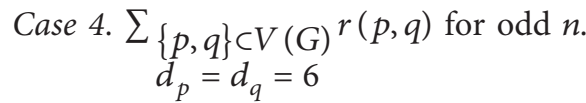

$$
\sum_{\substack{\{p, q\} \subset V \\ d_{p}=d_{q}=6}} d_{p} d_{q} r(p, q)=18 \sum_{t=1}^{((n-1) / 2)}\left(n t-t^{2}\right) .
$$

Case 5. $\sum\{p, q\} \subset V(G) \quad r(p, q)$ for even $n$.

$$
d_{p}=3, d_{q}=6
$$$$
\sum_{\{p, q\} \subset V} d_{p} d_{q} r(p, q)=18 \sum_{t=1}^{(n / 2)}\left(2 n t-2 t^{2}+2 t-\frac{1}{2}\right) \text {. }
$$$$
d_{p}=3, d_{q}=6
$$

Case 6. $\sum_{\substack{\{p, q\} \subset V(G) \\ d_{p}=3, d_{q}=6}} r(p, q)$ for odd $n$.

$\sum_{\substack{\{p, q\} \subset V \\ d_{p}=3, d_{q}=6}} d_{p} d_{q} r(p, q)=\frac{9 n^{2}+18 n}{2}+18 \sum_{t=1}^{((n-1) / 2)}\left(2 n t-2 t^{2}+2 t-\frac{1}{2}\right)$. 
If $n$ is even, through equations (24), (26), and (28) and Lemma 2, then

$$
\begin{aligned}
K f^{*}(C(n)) & =-\frac{9}{2} n+9 \sum_{t=1}^{(n / 2)}\left(8 n t-8 t^{2}+8 t-3\right) \\
& =-\frac{9}{2} n+9\left[-8 \sum_{t=1}^{(n / 2)} t^{2}+8 n \sum_{t=1}^{(n / 2)} t+\sum_{t=1}^{(n / 2)}(8 t-3)\right] \\
& =-\frac{9}{2} n+9\left[-8 \frac{(n / 2)((n / 2)+1)(n+1)}{6}+8 n \frac{(n / 2)((n / 2)+1)}{2}+\frac{(n / 2)(4 n+2)}{2}\right] \\
& =6 n^{3}+18 n^{2}-6 n .
\end{aligned}
$$

If $n$ is odd, through equations (25), (27), and (29) and Lemma 2, then

$$
\begin{aligned}
K f^{*}(C(n)) & =\frac{18 n^{2}+27 n-9}{2}+9 \sum_{t=1}^{((n-1) / 2)}\left(8 n t-8 t^{2}+8 t-3\right) \\
& =\frac{18 n^{2}+27 n-9}{2}+9\left[-8 \sum_{t=1}^{((n-1) / 2)} t^{2}+8 n \sum_{t=1}^{((n-1) / 2)} t+\sum_{t=1}^{((n-1) / 2)}(8 t-3)\right] \\
& =\frac{18 n^{2}+27 n-9}{2}+9\left[-8 \frac{((n-1) / 2)(((n-1) / 2)+1)(n-1+1)}{6}+8 n \frac{((n-1) / 2)(((n-1) / 2)+1)}{2}+\frac{((n-1) / 2)(4 n-2)}{2}\right] \\
& =6 n^{3}+18 n^{2}-6 n .
\end{aligned}
$$

This completes the proof.

3.2.3. Kemeny's Constant, Randic Index, and Zagreb Index. The relationship between vertices and edges of $C(n)$ based on degree is shown in Tables 4 and 5.

By utilizing equations (4)-(7) and Tables 4 and 5, the following three indices can be obtained easily:

$$
\begin{aligned}
M_{1}(C(n)) & =2 n \times 3^{2}+n \times 6^{2}=54 n, \\
M_{2}(C(n)) & =n \times(3 \times 3)+n \times(6 \times 6)+4 n \times(3 \times 6)=117 n, \\
R(C(n)) & =n \times \frac{1}{\sqrt{3 \times 3}}+n \times \frac{1}{\sqrt{6 \times 6}}+4 n \times \frac{1}{\sqrt{3 \times 6}} \\
& =\frac{(3+4 \sqrt{2}) n}{6} .
\end{aligned}
$$

If $n$ is even, then

$$
\begin{aligned}
K(C(n)) & =\frac{1}{4 \times 6 n}\left[-\frac{9}{2} n+9 \sum_{t=1}^{(n / 2)}\left(8 n t-8 t^{2}+8 t-3\right)\right] \\
& =\frac{n^{2}+3 n-1}{4} .
\end{aligned}
$$

If $n$ is odd, then

$$
\begin{aligned}
K(C(n)) & =\frac{1}{4 \times 6 n}\left[\frac{18 n^{2}+27 n-9}{2}+9 \sum_{t=1}^{((n-1) / 2)}\left(8 n t-8 t^{2}+8 t-3\right)\right] \\
& =\frac{n^{2}+3 n-1}{4} .
\end{aligned}
$$


TABLE 4: The classification of vertex-degree of $C(n)$.

\begin{tabular}{lcc}
\hline$d_{p}$ & 3 & 6 \\
\hline Number of vertices & $2 n$ & $n$ \\
\hline
\end{tabular}

TABLe 5: The classification of edge-degree of $C(n)$.

\begin{tabular}{lccc}
\hline$\left(d_{p}, d_{q}\right)$ & $(3,3)$ & $(6,6)$ & $(3,6)$ \\
\hline Number of edges & $n$ & $n$ & $4 n$ \\
\hline
\end{tabular}

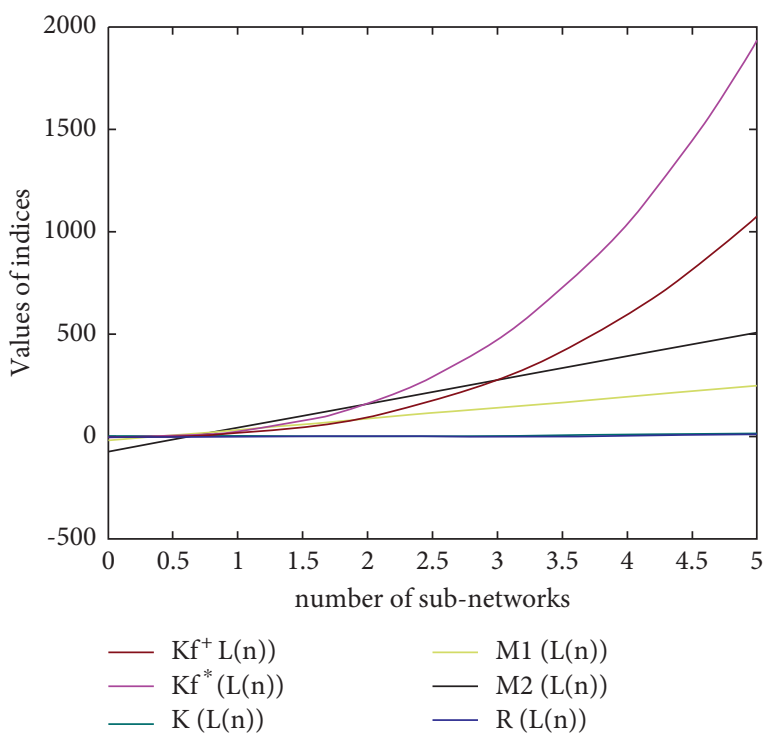

FIgURE 5: Comparisons of the topological indices for the linear tetrahedral network.

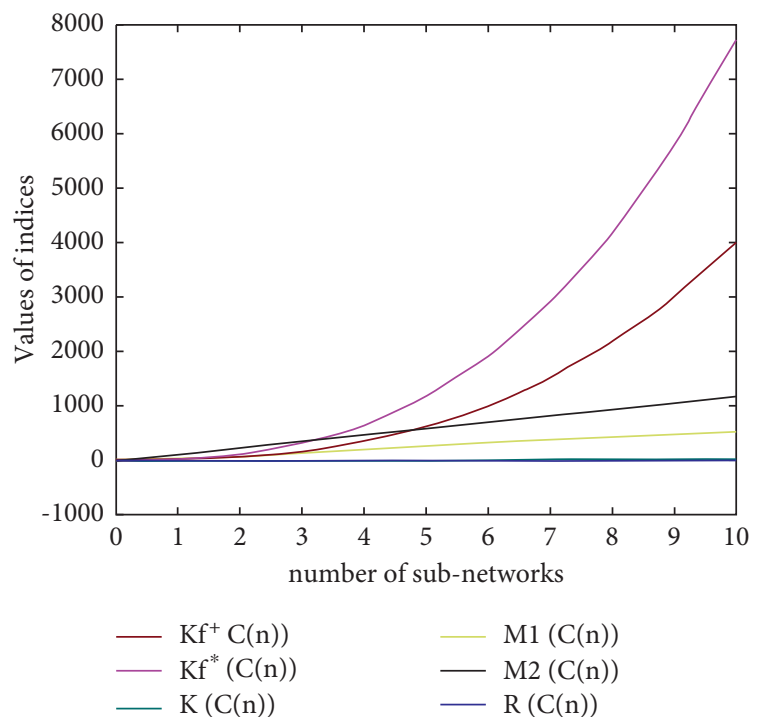

Figure 6: Comparisons of the topological indices for the circle tetrahedral network.
And the comparisons of the five topological indices obtained above are shown in Figures 5 and 6.

According to Figures 5 and 6, it is clear that the multiplicative degree-Kirchhoff index of both the linear and circle graph is the fast growing, and the additive degreeKirchhoff index is the second growing. Other than that, in the circle graph, whenever $n$ is odd or even, the Zagreb index is growing faster than Randic index and Kemeny's constant. And in the linear graph, the Zagreb index is also growing faster than Kemeny's constant and Randic index.

\section{Conclusion}

In this paper, two types of tetrahedral networks have been discussed and the resistance distance in different cases has been computed, respectively. Above all, the multiplicative (the additive) degree-Kirchhoff index of the networks has been calculated. Then, Kemeny's constant, Randic index, and Zagreb index have also been derived. Furthermore, the comparisons of the topological indices for the linear and circle networks have been studied. In future, we will devote ourselves to research more properties for types of stereochemical networks.

\section{Data Availability}

The figures, tables, and other data used to support this study are included within the article.

\section{Conflicts of Interest}

The authors declare that there are no conflicts of interest regarding the publication of this paper.

\section{Acknowledgments}

This work was supported in part by Anhui Provincial Natural Science Foundation under Grant 2008085J01 and Natural Science Fund of Education Department of Anhui province under Grant KJ2020A0478.

\section{References}

[1] Z. M. Li, Z. Xie, J. P. Li, and Y. G. Pan, "Resistance distancebased graph invariants and spanning trees of graphs derived from the strong prism of a star," Applied Mathematics and Computation, vol. 382, pp. 1-9, 2020.

[2] D. J. Klein and M. Randić, "Resistance distance," Journal of Mathematical Chemistry, vol. 12, no. 1, pp. 81-95, 1993.

[3] D. A. Spielman and N. Srivastava, "Graph sparsification by effective resistances," SIAM Journal on Computing, vol. 40, no. 6, pp. 1913-1926, 2011.

[4] H. Chen and F. Zhang, "Resistance distance and the normalized Laplacian spectrum," Discrete Applied Mathmatics, vol. 155, no. 5, pp. 654-661, 2017.

[5] I. Gutman, L. Fei, and G. Yu, "Degree resistance distance of unicyclic graphs," Transactions on Combinatorics, vol. 1, no. 2, pp. 27-40, 2012.

[6] R. van der Hofstad, T. Hulshof, and J. Nagel, "Random walk on barely supercritical branching random walk," Probability Theory and Related Fields, vol. 177, no. 1-2, pp. 1-53, 2020. 
[7] J. Engländer and S. Volkov, "Impatient random walk," Journal of Theoretical Probability, vol. 32, no. 4, pp. 2020-2043, 2019.

[8] Y. Li, Y. Wang, H. Lin, and T. Zhong, "First arrival time picking for microseismic data based on DWSW algorithm," Journal of Seismology, vol. 22, no. 4, pp. 833-840, 2018.

[9] X. Liao, J. Cao, J. Hu, J. You, X. Jiang, and Z. Liu, "First arrival time identification using transfer learning with continuous wavelet transform feature images," IEEE Geoscience and Remote Sensing Letters, vol. 17, no. 11, pp. 2002-2006, 2020.

[10] A. E. Kyprianou, V. Rivero, and B. Şengül, "Conditioning subordinators embedded in Markov processes," Stochastic Processes and their Applications, vol. 127, no. 4, pp. 1234-1254, 2017.

[11] A. Schnurr, "On deterministic Markov processes: expandability and related topics," Markov Processes and Related Fields, vol. 19, no. 4, pp. 693-720, 2013.

[12] Y. Ephraim and W. J. J. Roberts, "An EM algorithm for Markov modulated Markov processes," IEEE Transactions on Signal Processing, vol. 57, no. 2, pp. 463-470, 2009.

[13] F. Grabski, "Semi-Markov failure rates processes," Applied Mathematics and Computation, vol. 217, no. 24, pp. 99569965, 2011.

[14] R. Pinsky, “"Kemeny's constant for one-dimensional diffusions," Electronic Communications in Probability, vol. 24, pp. 1-5, 2019.

[15] R. E. Kooij and J. L. A. Dubbeldam, “Kemeny's constant for several families of graphs and real-world networks," Discrete Applied Mathematics, vol. 285, pp. 96-107, 2020.

[16] X. Wang, J. L. A. Dubbeldam, and P. Van Mieghem, "Kemeny's constant and the effective graph resistance," Linear Algebra and its Applications, vol. 535, pp. 231-244, 2017.

[17] R. Cruz, J. Monsalve, and J. Rada, "Trees with maximum exponential Randić index," Discrete Applied Mathematics, vol. 283, pp. 634-643, 2020.

[18] Y. Shi, "Note on two generalizations of the Randić index," Applied Mathematics and Computation, vol. 265, pp. 10191025, 2015.

[19] Z. Iqbal, A. Aslam, M. Ishaq, and W. Gao, "The edge versions of degree-based topological descriptors of dendrimers," Journal of Cluster Science, vol. 31, no. 2, pp. 445-452, 2020.

[20] S. M. Kang, W. Nazeer, M. A. Zahid, A. R. Nizami, A. Aslam, and M. Munir, "M-polynomials and topological indices of hex-derived networks," Open Physics, vol. 16, no. 1, pp. 394-403, 2018.

[21] J.-B. Liu, C. Wang, S. Wang, and B. Wei, "Zagreb indices and multiplicative Zagreb indices of Eulerian graphs," Bulletin of the Malaysian Mathematical Sciences Society, vol. 42, no. 1, pp. $67-78,2019$.

[22] B. Furtula, A. Graovac, and D. Vukičević, "Augmented Zagreb index," Journal of Mathematical Chemistry, vol. 48, no. 2, pp. 370-380, 2010.

[23] M. S. Sardar, X. F. Pan, and S. A. Xu, "Computation of resistance distance and Kirchhoff index of the two classes of silicate networks," Applied Mathematics and Computation, vol. 381, pp. 1-10, 2020. 